\title{
Procedimiento constructivo de muros de sótano mediante bataches con juntas de conexión. Estudio del ancho óptimo de excavación en suelos mixtos
}

\section{Construction procedure of basement walls using staged bermed excavation with connection joints. Study of the optimum excavated berm section in mixed soils}

$\underline{\text { M. Cano }}^{(*)}$, J.L. Pastor ${ }^{(*)}$, T. Miranda ${ }^{(* *)}$, R. Tomás ${ }^{(*)}$

RESUMEN

La construcción de muros de sótano mediante bataches es una técnica ampliamente empleada en muchos países, sin embargo, no existe normativa que la regule, ni estudios científicos que avalen su uso. Con el fin de aumentar la productividad, la tendencia en la ejecución de este tipo de construcciones es realizar las excavaciones parciales con las máximas dimensiones posibles, que deben incluir el espacio necesario para solapar las armaduras horizontales. En este trabajo se presenta un procedimiento de ejecución de los muros con juntas de conexión, sin solape de armaduras horizontales y se modeliza mediante un programa de elementos finitos con el fin de obtener el ancho de excavación óptimo para suelos mixtos. Los resultados avalan la mejora en la estabilidad de esta técnica de ejecución y revelan una relación lineal entre la cohesión y el ancho de excavación para un ángulo de rozamiento determinado.

Palabras clave: Muro de sótano, excavación por bataches, junta de conexión, factor de seguridad, elementos finitos, suelos mixtos.

\section{ABSTRACT}

The construction of basement walls using staged berm is a widely applied technique in many countries. However, there are neither regulation nor scientific studies to support its use. In order to increase productivity, the tendency in the execution of this type of constructions is to carry out the partial excavations with the maximum possible dimensions, which must include the necessary space to overlap the horizontal reinforcements. This paper presents a procedure for the execution of walls with connection joints, without overlapping horizontal reinforcements and is modeled by a finite element software in order to obtain the optimum excavation width for mixed soils. The results underline the improvement in the stability of this execution technique and reveal a linear relationship between cohesion and excavation width for a given friction angle.

Keywords: Basement wall, staged bermed excavation, connection joint, factor of safety, finite elements, mixed soils.

(*) Departamento de Ingeniería Civil. Escuela Politécnica Superior, Universidad de Alicante, Alicante (España).

(**) ISISE - Departamento de Engenharia Civil. Universidade do Minho, Guimarães (Portugal).

Persona de contacto/Corresponding author: miguel.cano@ua.es (M. Cano).

ORCID: https://orcid.org/oooo-0002-5737-9299 (M. Cano); https://orcid.org/oooo-0oo1-7870-3652 (J.L. Pastor); https://orcid.org/oooo-0003-4054-686o (T. Miranda); https://orcid.org/oooo-0oo3-2947-9441 (R. Tomás).

Cómo citar este artículo/Citation: Cano, M.; Pastor, J.L.; Miranda, T.; Tomás, R. (2020). Procedimiento constructivo de muros de sótano mediante bataches con juntas de conexión. Estudio del ancho óptimo de excavación en suelos mixtos. Informes de la Construcción, 72(558): e344. https://doi.org/10.3989/ic.66913.

Copyright: (C) 2020 CSIC. Este es un artículo de acceso abierto distribuido bajo los términos de la licencia de uso y distribución Creative Commons Reconocimiento 4.o Internacional (CC BY 4.0). 


\section{INTRODUCCIÓN}

En terrenos con el nivel freático elevado, los métodos de vaciado de un solar están basados frecuentemente en creación de un recinto estanco que a su vez es el elemento estructural definitivo que retiene el empuje del terreno. De entre ellos se destaca la construcción de los denominados muros-pantalla in situ (diaphragm wall, en inglés). En esta técnica constructiva, la excavación se realiza desde la superficie del solar, en forma de zanja y por tramos, hasta alcanzar la profundidad total de la pantalla. En el caso de que el nivel freático durante la fase constructiva esté por debajo del plano de cimentación, se puede optar también por la construcción de muros discontinuos de pilotes o de micropilotes, que se adaptan mejor a solares de difícil acceso de maquinaria pesada. Estas modalidades constructivas permiten un vaciado del solar con las máximas garantías de seguridad para los operarios, así como para los bienes y servicios que limitan con el área de actuación. La limitación de los desplazamientos y la estabilidad del muro de contención se consiguen mediante la introducción de anclajes activos y/o mediante la colocación de codales a medida que se profundiza en el vaciado del solar.

Tan solo cuando las edificaciones y los servicios públicos están alejados de los límites de parcela donde se pretende ejecutar el vaciado y las características geotécnicas del terreno lo permitan, se podrá optar por una excavación completa del solar sin elementos de contención provisionales. No obstante, existe una situación intermedia en la que la excavación del perímetro del solar se puede realizar en etapas. Este sistema está basado en la excavación alterna de tramos del frente de una berma perimetral previamente ejecutada. En España, este tipo de excavación recibe el nombre de excavación por bataches y tan solo es ejecutable si el nivel freático permanece por debajo del plano de cimentación durante la etapa constructiva y el terreno tiene la suficiente cohesión como para sostenerse entre las bermas laterales, que trabajan a modo de contrafuertes, durante la etapa de excavación de cada batache (Figura 1). Los bataches suelen emplearse cuando la excavación es de un solo sótano, aunque en ocasiones también se ejecutan excavaciones con este sistema con 2 ó 3 sótanos. Si se trata de dos sótanos se puede optar por la excavación mediante bataches de 2 sótanos de altura o mediante un sistema más complejo basado en la creación de anillos descendentes, generalmente anclados, opción empleada habitualmente en el caso de tres sótanos. Hay que hacer notar que cada uno de estos anillos se excava también en distintas etapas, dejando bermas laterales, es decir, la excavación es igualmente por bataches (Figura 1c).

El vaciado de un solar empleando la técnica de excavación mediante bataches es una técnica ampliamente empleada, tanto en España como en otros países. En algunos casos este sistema de excavación viene impuesto por las dimensiones del solar, que dificultan la entrada de maquinaria específica para la construcción de un sostenimiento previo a su excavación. Otras veces, aunque la solución mediante procedimientos más seguros sea factible, no es una alternativa económicamente viable por las dimensiones de la construcción, sobre todo si se trata de un único sótano.

Con el fin de aumentar la productividad, la tendencia en la ejecución de excavaciones mediante bataches es realizar éstos con unas dimensiones acordes con las de las dimensiones de las placas de encofrado existentes en el mercado, y con un ancho lo mayor posible. Sin embargo, la excavación del batache debe ser algo superior a la dimensión del elemento hormigonado, con objeto de permitir la presencia de las esperas de las armaduras horizontales, pues la conexión lateral es necesaria según las normativas técnicas (e.g. Ministerio del a Vivienda, 2008) (1). Este exceso de excavación suele ser de aproximadamente $60 \mathrm{~cm}$ en cada lateral y como mínimo alrededor de $20-30 \mathrm{~cm}$, en el caso de que se opte por doblar las armaduras. Es decir, la exis-
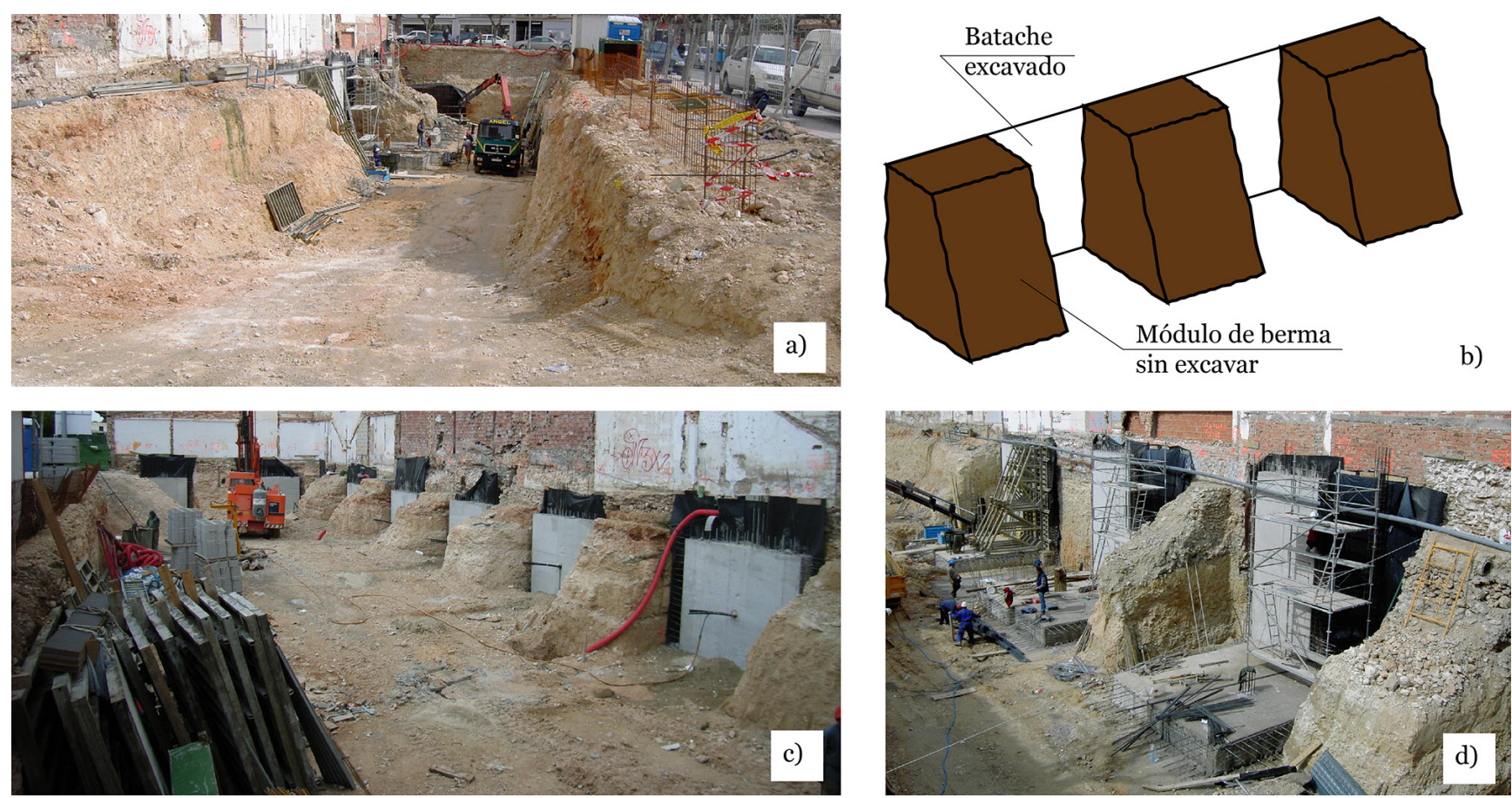

Figura 1. Fases del proceso de construcción de un muro de un sótano por bataches: a) Excavación central del solar dejando bermas continuas en los laterales, b) esquema del proceso de excavación parcial de la berma, c) ejecución y anclaje de módulos de muro en los bataches excavados para la ejecución de anillos descendentes y d) ejecución de módulos de muro autoestables (incluido cimentación) en los bataches excavados. 
tencia de estos sobreanchos conlleva a que los valores de la excavación abierta se incrementen de forma considerable, con el consiguiente riesgo de inestabilidad del frente excavado. Por ejemplo, en circunstancias normales, un batache de $2 \mathrm{~m}$ de ancho, compatible con las propiedades geotécnicas medias de un terreno con una baja cohesión, necesita alrededor de $3 \mathrm{~m}$ para ser operativo, lo cual aumenta la probabilidad de ocurrencia de inestabilidades durante la fase constructiva (Figura 2).

Adicionalmente, la conexión entre bataches a través de la armadura horizontal genera otros problemas, tales como el desdoblado, generalmente mal ejecutado, si es que se opta por esta solución. Además, como es habitual en cualquier obra en contacto con el terreno, las armaduras quedan sucias, con restos de tierra, sobre todo si el terreno presenta cierta humedad. Por otra parte, las juntas de hormigonado entre bataches son muy difíciles de ejecutar, tanto por su irregularidad como por la posterior excavación e intrusión de terreno entre el hormigón. Ello conduce a que la unión no sea continua y que, tras la obra, dicha junta comprometa la estanqueidad del sótano terminado.

Una vez que se ha ejecutado el panel de hormigón armado correspondiente a un batache, debe asegurarse su estabilidad provisional hasta la ejecución del sostenimiento definitivo. Esta estabilidad provisional se consigue mediante anclajes al terreno, mediante acodalamientos o haciendo autoestable el conjunto muro-cimiento. En la práctica habitual, los paneles son de hormigón armado y ejecutados in situ, aunque existen patentes de ejecución mediante paneles prefabricados (2).

A pesar del extendido empleo de esta técnica constructiva, sin embargo, no existe ninguna normativa que la regule, ni tampoco estudios científico-técnicos que validen su empleo. En España, tan solo las antiguas Normas Tecnológicas de la Edificación, NTE, del Ministerio de la Vivienda (3), ya en desuso, hacían referencia al ancho de excavación, aunque de forma genérica y sin tener en cuenta los parámetros geotécnicos del terreno. En España y en Portugal se admiten reglas sancionadas por la práctica en función del comportamiento observado por el terreno.

La norma británica BS6031 (4) proporciona reglas y recomendaciones para los movimientos de tierra que abarcan terraplenes y excavaciones, la nivelación y la compactación, etc. En ella, el método de excavación más asimilable a lo que se entiende por excavación por bataches es el denominado "dumpling method", que consiste en construir una serie de muros de contención en una trinchera alrededor del perímetro del solar, sección a sección (bataches), dejando un centro llamado "dumpling". Los trozos de trinchera excavados deben ser estabilizados por sistemas tipo método berlinés o tablestacas en caso de terrenos poco resistentes. A continuación, se confeccionan los paneles de muros de hormigón, que deben estabilizarse convenientemente y cuando éstos se han ejecutado, comienza la excavación en el centro en franjas. Se recomienda su utilización para profundidades de excavación de un máximo de $3 \mathrm{~m}$ (1 sótano) y respecto del ancho solo hay una limitación de $15 \mathrm{~m}$ si el trozo se sostiene mediante un sistema tipo método berlinés.

Este déficit de publicaciones sobre esta técnica constructiva, sin embargo, contrasta con los numerosos trabajos realiza- dos sobre el empleo de bermas como sostenimiento provisional de muros pantalla (e.g. Clough and Denby, 1977 (5), Powrie et al., 1992 (6), Gourvenec and Powrie, 2000 (7), Gomaa, 2003 (8)). De hecho, este método constructivo puede ser un método económico para la construcción de sótanos en áreas urbanas si el movimiento del suelo alrededor de la excavación no es un factor determinante y el espacio de la excavación es amplio (9). Como ejemplo, cabe destacar las conclusiones del trabajo de Gourvenec y Powrie (7) que establecen que el movimiento de la pantalla es linealmente dependiente del ancho de la berma excavada y, una vez fijado un ancho límite, dicho movimiento depende no solo de dicho ancho, sino también de la distancia entre bataches excavados. Además, todos estos autores coinciden en resaltar en sus análisis realizados mediante modelización numérica la importancia de tener en cuenta el efecto tridimensional en este tipo de excavaciones.

Por otra parte, hay trabajos que hacen referencia a la estabilidad de los paneles de muro pantalla considerando el hueco de la excavación, que también pueden considerarse como un tipo de batache $(10,11)$. En esta misma línea de investigación también hay referencias de estudios similares respecto del comportamiento tensional en excavaciones de huecos rectangulares artificiales (12). Aunque los resultados obtenidos también tienen una gran influencia tridimensional, el comportamiento tenso-deformacional del terreno es diferente al de una berma excavada por bataches.

En este trabajo se describe un procedimiento constructivo para la ejecución de muros de sótano por bataches que consiste en la ejecución de una junta de conexión encofrada, de forma cóncavo-convexa o poligonal en los laterales de los paneles hormigonados, a la que se le añade un cordón hidrófilo. De esta manera se eliminan las esperas de las armaduras horizontales y la unión de los diferentes módulos de bataches se confiere al encaje mecánico entre paneles, debido a su forma. Por otra parte, al igual que sucede con la ejecución tradicional de los muros-pantalla, se ejecuta una viga de coronación en cabeza que obliga a un trabajo conjunto de los módulos. Basado en este procedimiento constructivo se realiza un análisis de la estabilidad de estos elementos durante la fase de excavación. El estudio se realiza para una geometría adaptada a la construcción de un sótano estándar y un suelo mixto, con una cohesión de entre moderada a baja (Figura 2).

Los objetivos principales que se persiguen con el empleo de esta técnica son, optimizar al máximo el ancho de la excavación con el fin de mejorar la seguridad y las condiciones de estanqueidad del recinto, al poder ejecutar juntas de mayor calidad con la incorporación de un cordón hidrófilo, que evitaría la eventual entrada de agua durante la vida útil de la estructura. Por otra parte, el estudio de estabilidad permitirá determinar el ancho del batache máximo para unas determinadas condiciones geotécnicas, con un nivel de seguridad adecuado.

Como se desprende de lo anteriormente comentado, tanto por la tipología constructiva analizada como por la manera de abordar la estabilidad de la excavación, el trabajo propuesto representa una novedad objetiva en el campo de la ingeniería geotécnica. Además, la repercusión técnica práctica en este campo también es importante, pues numerosas excavaciones que se realizan mediante este sistema 

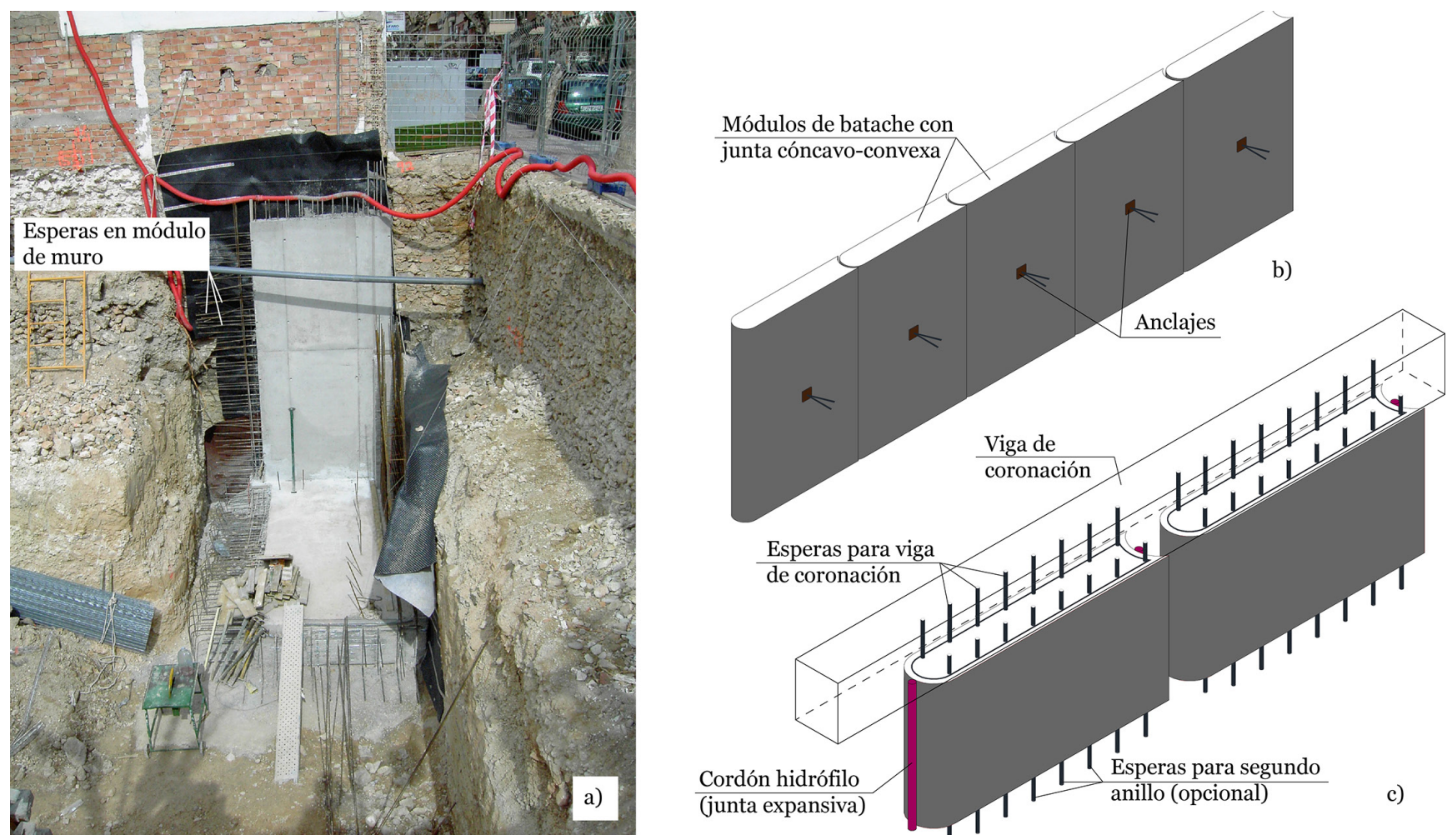

Figura 2. Detalle de un batache tradicional con un módulo de muro autoestable en el que se observan las esperas de conexión horizontales (a) y esquema de la metodología constructiva analizada en este trabajo (b y c). Nótese que los módulos de batache mostrados en (c) se han representado separados para una mejor visualización.

se basan en criterios de experiencia local, sin ningún criterio científico objetivo y los resultados no son siempre satisfactorios. Ello conlleva un elevado riesgo para la seguridad en el trabajo y de los bienes y servicios del entorno de la obra.

\section{METODOLOGÍA}

\subsection{Procedimiento constructivo}

El procedimiento constructivo aquí presentado pretende utilizar las ventajas de la técnica de construcción de los murospantalla en la construcción de los muros mediante bataches, con lo que se logra que la excavación tenga estrictamente el ancho del panel a hormigonar en cada fase. Además, mediante la inclusión de un cordón hidrófilo o junta impermeable en la conexión entre los bataches se pretende mejorar las condiciones de estanqueidad de los sótanos, que son poco satisfactorias en cualquiera de las técnicas habituales. No obstante, hay que tener en cuenta que este tipo de excavaciones solo se puede llevar a cabo si el nivel freático está por debajo del plano de soporte de la cimentación. Por esta razón, no cabe esperar un gran flujo de agua en la fase de explotación del edificio, salvo en situaciones extraordinarias, tales como inundaciones, fugas de agua, etc. Por tanto, no es necesario colocar una junta elastomérica anclada en el hormigón. El cordón hidrófilo propuesto es expansivo, bien de composición bentonítica o bien con polímeros expansivos y se fija mediante un adhesivo sobre la superficie del hormigón ya desencofrada en el centro de la cara cóncava o convexa. Además, la propia morfología de la junta dificulta la entrada de agua al recinto. En consecuencia, con el sistema propuesto los bataches se ejecutarán con una junta especial encofrada, de forma cóncavoconvexa en los laterales, a la que se le añade un cordón hidró- filo. De esta manera se eliminan las esperas de las armaduras horizontales. La unión de los diferentes módulos de bataches se confiere al contacto especial entre paneles, debido a su forma. Por otra parte, al igual que sucede con la ejecución tradicional de los muros-pantalla, se ejecuta una viga de coronación en cabeza que asegura el trabajo conjunto de los módulos (Figura 2).

Tal y como ya se ha mencionado, si la excavación del batache se realiza según la metodología tradicional, éste debe ser algo superior al ancho total del encofrado, con objeto de permitir la presencia de las esperas de las armaduras horizontales, quedando normalmente la armadura con restos de tierra y en ocasiones, doblados debido a la maquinaria de excavación. Por otra parte, la existencia de los sobreanchos conlleva a que los valores de excavación abierta se incrementen de forma considerable, con el consiguiente riesgo de inestabilidad del frente excavado. Por último, la posible existencia en la excavación de terrenos con cierta humedad provocará, por un lado, que las armaduras se encuentren extremadamente sucias y con una difícil puesta en obra, y por otro, que las juntas de hormigonado no se encuentren completamente limpias, siendo permeables al paso de agua una vez hormigonadas.

En este trabajo se han modelizado bataches de anchos 2.7, 3.0, 3.4, 3.7, 4.0 y 5.0 m, siendo todos ellos combinación de los distintos anchos de las placas de encofrado metálico más habituales en el mercado y con el fin de validar la bondad del método constructivo. Algunos de estos anchos de excavación se corresponden con el ancho del encofrado más el exceso de excavación considerado para las armaduras de conexión, que será de $100 \mathrm{~cm}$ en el caso de prolongación recta y de $40 \mathrm{~cm}$ en caso de que se doblen. 
La combinación de placas utilizadas da unos anchos de encofrado de 2.7, 3.0 y $4.0 \mathrm{~m}$, que en ocasiones coincidirán con el ancho de excavación si se emplea la técnica propuesta. En caso contrario el ancho será mayor.

Respecto a la profundidad de la excavación cabe comentar que se ha tomado una altura total de excavación del batache tipo de $3.5 \mathrm{~m}$, que es el resultado de considerar una excavación de $10 \mathrm{~cm}$ para el hormigón de limpieza, $60 \mathrm{~cm}$ para el canto de la cimentación, 2.5 m para la altura del sótano y 0.3 $\mathrm{m}$ para el canto de forjado, dimensiones medias todas ellas habituales en edificación.

\subsection{Suelos mixtos}

A menudo, los suelos presentan una estructura formada por una mezcla de partículas cohesivas y granulares. Estos suelos se denominan suelos intermedios o mixtos y se encuentran ampliamente representados tanto en depósitos naturales como en estructuras de tierra hechas por el hombre $(13,14)$.

Aunque en realidad en este trabajo se realiza un estudio paramétrico y por lo tanto generalizable a todo tipo de suelos, con el fin de adoptar valores de los parámetros geotécnicos reales, se han seleccionado suelos de referencia limo-arcillosos con inclusiones de arenas y/o gravas de la provincia de Alicante (España) y suelos residuales (jabres) del norte de Portugal. Los parámetros geotécnicos se han obtenido a partir de numerosos estudios geotécnicos y de publicaciones científicas sobre estos materiales.

Los suelos residuales muestran un comportamiento mecánico específico diferente de los establecidos para los suelos transportados como sedimentos, ya que la presencia de una matriz cementada juega un papel importante en la resistencia y el comportamiento de rigidez, especialmente a poca profundidad (baja tensión de confinamiento). Además, la ausencia de nivel freático por encima de la cota de cimentación genera fenómenos de succión, que también reportan una influencia significativa en las propiedades de resistencia y rigidez (15).

Es importante hacer notar que los jabres que se han tenido en cuenta en este trabajo son suelos residuales en sentido estricto, es decir, no queda ningún vestigio de la estructura original de la roca. Los ensayos realizados por Viana da Fonseca et al (16) en dos enclaves de jabres de Braga indicaban que $c^{\prime}=0-3 \mathrm{kPa}$ y $\Phi^{\prime}=32^{\circ}-41^{\circ}$ y c' $=4-46 \mathrm{kPa}$ y $\Phi^{\prime}=25^{\circ}-32^{\circ}$. Por su parte, Cruz et al (17), determinaron para suelos de Vila do Conde el siguiente par de valores: $c^{\prime}=11, \Phi^{\prime}=35$. Adicionalmente, Cruz (18), tras la revisión de trabajos previos, concluyó que como valores promedios para suelos residuales de la zona norte de Portugal se puede adoptar una cohesión con un rango de valores de entre 5 y $30 \mathrm{kPa}$, un ángulo de rozamiento interno de entre $35^{\circ}$ y $38^{\circ}$ y un peso específico de entre 17.0 y $19.0 \mathrm{kN} / \mathrm{m}^{3}$.

Respecto de los suelos mixtos de Alicante, cabe comentar que se trata de depósitos correspondientes a sedimentos de origen aluvial, formados por gravas, arenas, limos y arcillas. Se trata pues, de suelos muy heterogéneos constituidos por gravas calcáreas con contenido variable en arenas, limos y arcillas intercalados por niveles arcillo-limosos con cierto contenido en arenas.
Las propiedades geotécnicas de estos suelos, así como la descripción realizada anteriormente se ha obtenido de diversos estudios geotécnicos. La alta heterogeneidad de estos suelos hace que estos valores presenten rangos muy amplios. Así el porcentaje de gravas varía entre un $5 \%$ y un $60 \%$, el de arenas entre un $15 \%$ y un $60 \%$ y el de finos (fracción que pasa por el tamiz $0.08 \mathrm{~mm}$ ) entre un $15 \%$ y un $95 \%$. Respecto a los parámetros geotécnicos, cabe decir que los valores de la cohesión y de ángulo de rozamiento interno son c' $=0-19 \mathrm{kPa}$ y $\Phi^{\prime}=25^{\circ}-38^{\circ}$. El peso específico seco oscila entre 16 y $18 \mathrm{kN} / \mathrm{m}^{3}$ y el peso específico aparente entre 18 y $21 \mathrm{kN} / \mathrm{m}^{3}$.

Además, en este tipo de suelos se da la circunstancia que, debido a las condiciones de sedimentación, la aridez del clima y la presencia de niveles freáticos profundos se producen fenómenos de succión capilar y/o cementación debido a cristalización de sales solubles. No obstante, este comportamiento favorable no se ha tenido en cuenta en el estudio, pues ambos fenómenos se ven condicionados por el estado de saturación del suelo.

Como se puede inferir de lo anteriormente expuesto, a pesar de haber elegido unos suelos muy particulares, las propiedades geotécnicas que presentan son representativas de un amplio abanico de suelos, por lo que los resultados de este trabajo son perfectamente extrapolables a otros tipos de terrenos que presenten parámetros geotécnicos dentro de los extensos límites aquí analizados.

\subsection{Análisis de la estabilidad del elemento excavado}

Los métodos de cálculo de la estabilidad de taludes más empleados en la práctica profesional son los métodos de equilibrio límite, basados en definir previamente un mecanismo de rotura y establecer el equilibrio de fuerzas o momentos para obtener las fuerzas movilizantes. Del modelo constitutivo del material se obtiene la fuerza que resiste el material en el plano de rotura. Finalmente, el Factor de Seguridad (FS) se obtiene comparando las fuerzas o momentos resistentes con los movilizantes. Por otra parte, los métodos numéricos discretizan el dominio del suelo y calculan los valores de las variables del problema en puntos de este dominio de manera que satisfagan las condiciones de equilibrio, compatibilidad, ley constitutiva y condiciones de contorno. De entre estos métodos destacan los métodos de elementos finitos (MEF), en los cuales, empleando el método de reducción de la resistencia al corte (c- $\Phi$ reduction), se puede obtener el factor de seguridad del mecanismo de rotura más desfavorable sin necesidad de definirlo a priori (19).

Para establecer la repercusión del ancho de la excavación parcial de la berma (batache) en su estabilidad, se debe considerar el efecto tridimensional (3D). En la actualidad el método de los elementos finitos permite analizar modelos $3 \mathrm{D}$, habiéndose convertido en una herramienta clave en este tipo de análisis.

Una de las dificultades del trabajo estriba en establecer el factor de seguridad adecuado (FS) para este tipo de sistema constructivo, pues tal y como se ha comentado anteriormente no existe normativa ni recomendaciones técnicas publicadas al respecto. Razón por la cual, se ha optado por consultar normativas y guías técnicas de situaciones de proyecto similares a ésta, tales como taludes o muros. 
La guía de cimentaciones en obras de carretera, GCOC, del Ministerio de Fomento (20), establece que para el coeficiente de seguridad total mínimo normal frente a la inestabilidad global de muros para la combinación de acciones casi permanente, en situaciones transitorias y de corto plazo, puede adoptarse el valor $\mathrm{FS}=1.3$. Sin embargo, el coeficiente de seguridad mínimo reducido es $\mathrm{FS}=1.2$, siendo de aplicación únicamente en construcciones provisionales, tales como las que son objeto de este estudio.

El Eurocódigo 7 utiliza coeficientes de seguridad parciales y para estudiar la estabilidad global de un talud en condiciones drenadas establece que $\gamma_{\mathrm{G}}=1$ y $\gamma_{\mathrm{c}^{\prime}}=\gamma_{\Phi}=1.25$, tomando como enfoque de proyecto (Design Approches) la situación de proyecto DA1.C2, que normalmente gobierna el comportamiento del talud, es decir, las acciones mantienen su valor representativo y se minoran los parámetros geotécnicos $(21,22)$.

Por su parte la AASHTO (23), en situación estática, para un talud provisional y sin cargas en la proximidad de su coronación, establece que, para reconocimientos geotécnicos que proporcionan valores fiables, puede emplearse un factor de seguridad de 1,33 .

El Soil Mechanics DESIGN MANUAL 7.01 de la NAVFAC (24) considera un coeficiente de seguridad global para un talud de entre 1.3 y 1.25 también en situaciones estáticas y para un talud provisional.

En el ámbito de la normativa minera, donde los taludes son siempre provisionales por definición y los factores de seguridad más ajustados, los coeficientes de seguridad adoptados son de 1.2 (25).

Teniendo en cuenta todo lo anteriormente expuesto, y considerando que el modelo constructivo no es estrictamente un talud, sino que, debido a la forma de la excavación, esta presenta un comportamiento resistente tridimensional y por tanto una mayor seguridad, se ha considerado adecuado tomar como factor de seguridad 1.2 en este trabajo, el cual viene refrendado por lo establecido en la GCOC (20). Este es el factor de seguridad con el que se afectará a los parámetros geotécnicos resistentes, factor de seguridad global que es asimilable a tomar ese mismo factor como coeficiente de seguridad parcial para minorar tanto a los términos “tg $\Phi$ " y “c” de la ecuación de Mohr-Coulomb.

El método de reducción de la resistencia al corte para obtener el factor de seguridad en un determinado modelo consiste en minorar la cohesión y el ángulo de rozamiento interno por un determinado FS, tomando éstos como los parámetros geotécnicos del modelo. El proceso se repite hasta que el modelo rompe. Cuando esto sucede, el FS del caso estudiado será el inmediatamente anterior al que produce la rotura. Sin embargo, en este trabajo, el procedimiento es algo diferente, pues considerando que $\mathrm{FS}=1.2$, se ha minorado la fricción y un valor inicial de la cohesión, repitiendo el procedimiento hasta rotura. En ese momento el valor de la cohesión inmediatamente anterior a la rotura y $\Phi$ ', mayorados por 1.2 serían los parámetros geotécnicos que permitirían un $\mathrm{FS}=1.2$ para un ancho determinado de batache. Es decir, para un ancho y $\Phi$ ' determinados se calcula la cohesión mínima que debe tener un suelo mixto para tener un $\mathrm{FS}=1.2$, lo cual se ha considerado realizar de esta manera, al observar en los tanteos previos que la cohesión era el parámetro que más influía en el FS.
En general, este tipo de suelos presentan un ángulo de rozamiento interno superior a $25^{\circ}$, aunque por razones de seguridad y con el fin de no dejar ningún caso sin tratar, se ha tomado como valor inferior de este estudio $20^{\circ}$, abarcando, además el rango de valores considerados todas las situaciones reales de este tipo de suelos. Por otra parte, la cohesión medible en ensayos triaxiales o de corte directo no suele ser alta, aunque fenómenos tales como la succión capilar o la cementación proporcionan comportamientos mejores que los esperados. No obstante, es aconsejable no tener en cuenta esta mejora, pues situaciones de saturación repentina pueden mermar o incluso anular este efecto favorable para la estabilidad.

Asimismo, en este estudio se ha utilizado un único peso específico aparente, siendo éste el valor más representativo de los suelos estudiados $\left(\gamma=19 \mathrm{kN} / \mathrm{m}^{3}\right)$. No se ha tenido en cuenta las condiciones de saturación repentina por agua de lluvia, al tratarse de una situación accidental, que se asumiría por el FS. Para cada uno de los anchos de batache modelizados (i.e. 2.7, 3.0, 3.4, 3.7, 4.0 y 5.0 m) se ha calculado un modelo considerando distintos valores de ángulo de rozamiento interno (i.e. $20^{\circ}, 25^{\circ}, 30^{\circ}, 35^{\circ}$ y $38^{\circ}$ ). En total se han realizado 35 simulaciones, llevando a cabo para cada una de ellas entre cuatro y cinco cálculos.

Los valores de los parámetros geotécnicos se han considerado en tensiones efectivas, pues a pesar de que este tipo de procedimiento constructivo es rápido, los suelos presentan una importante fracción granular y presentar un bajo grado de saturación.

Asimismo, no se consideran en este estudio cargas en coronación, ni la presencia de nivel freático por encima de la cota inferior de excavación, atendiendo a las recomendaciones de las antigua Normas Tecnológicas del Ministerio de la Vivienda (3). En estos casos se considera que debe recurrirse a otros procedimientos constructivos con la incorporación de un sostenimiento previo a la excavación.

Tampoco se han considerado las acciones sísmicas, al tratarse de una acción accidental de poca probabilidad de ocurrencia dado que la ejecución de este procedimiento de construcción es rápida.

El análisis mediante el método de elementos finitos se ha realizado utilizando el programa PLAXIS 3D (v2.o). El modelo constitutivo elegido para estudiar la rotura de la excavación es el de Mohr-Coulomb. En este sentido el modelo MohrCoulomb predice de forma apropiada el fallo al corte de los suelos en condiciones drenadas (26). De la misma forma, Pots and Zdravkovic (27) indican que los modelos constitutivos elásticos - perfectamente plásticos, como el Mohr-Coulomb, pueden ser empleados satisfactoriamente en la modelización de excavaciones en suelos de baja plasticidad.

La versión del programa PLAXIS con la que se ha realizado este estudio no permite definir las bermas en forma de plano inclinado, es decir, el perfil del talud ha de definirse de manera escalonada, siguiendo el mismo procedimiento que el empleado por otros autores como Segato y Scarpelli (28) para modelizar taludes inclinados. En general, los escalones se han definido de $50 \mathrm{~cm}$, aunque en uno de los modelos los escalones se han tomado de $1 \mathrm{~m}$ de ancho para evitar roturas localizadas en la modelización numérica. No obstante, esta modificación de la geometría no afecta a la modelización rea- 
lizada pues se estudia la rotura de la pared vertical del batache y no de la berma (Figura 4a).

En todos los modelos considerados en el estudio se ha establecido un mismo ancho para los módulos excavados y sin excavar. Las etapas constructivas modelizadas para la formación de módulo de berma se corresponden a una excavación escalonada de $50 \mathrm{~cm}$ de huella y contrahuella descendente hasta alcanzar la cota -3.50, que es la profundidad de excavación justificada anteriormente, siendo siempre el frente vertical en el módulo excavado (Figura 4a).

Asimismo, cabe comentar que el mallado de los elementos finitos en la parte central está más refinado, con el fin de obtener resultados más precisos.

\section{RESULTADOS}

Los resultados muestran una clara tendencia lineal en el aumento de la cohesión necesaria para alcanzar el FS=1.2 a medida que el ángulo de fricción disminuye (Tabla 1). Esta tendencia lineal se refleja en los resultados con rectas de ajuste que presentan un coeficiente de determinación $\left(\mathrm{R}^{2}\right)$ de $0.960-$ 0.997. Su pendiente es similar para los anchos de batache 2.7, 3.0, 3.4, 3.7 y $4.0 \mathrm{~m}$, con valores que oscilan entre -0.18 y -0.21. Sin embargo, para el batache de mayor ancho $(5 \mathrm{~m})$ dicha pendiente aumenta ligeramente (-0.27) (Figura 3a).

Por otra parte, para el menor de los ángulos de rozamiento estudiados (i.e. $2^{\circ}$ ) existe también una tendencia marcada lineal creciente en los valores de cohesión necesarios para alcanzar un FS=1.2 al ir aumentado el ancho del batache excavado $\left(\mathrm{R}^{2}=0.99\right)$. Para el ángulo de $25^{\circ}$, sin embargo, esta tendencia se suaviza al final y el ajuste lineal ya no es tan bueno $\left(R^{2}=0.91\right)$, presentado un mejor ajuste $\left(R^{2}=0.97\right)$. Por otra parte, para ángulos de rozamiento mayores (i.e. $30^{\circ}, 35^{\circ}$ y $38^{\circ}$ ) esta tendencia se atenúa aún más a medida que el ancho de batache se hace mayor, por lo que la tendencia general es claramente cuadrática (Figura 3b).

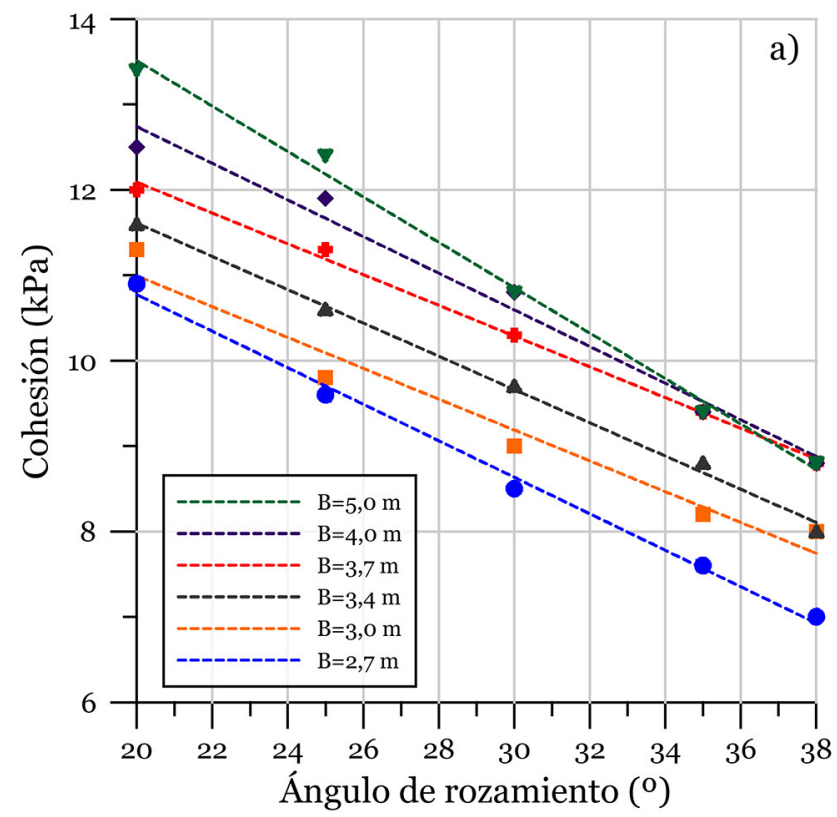

Tabla 1. Resultados de la modelización. Valores de cohesión $(\mathrm{kPa})$ necesarios para un FS=1.2 en función del ancho de batache y del ángulo de rozamiento interno.

\begin{tabular}{|c|c|c|c|c|c|}
\hline \multirow{2}{*}{$\begin{array}{c}\text { Ancho de } \\
\text { excavación B (m) }\end{array}$} & \multicolumn{5}{|c|}{$\boldsymbol{\Phi ( \mathbf { ) }}$} \\
\cline { 2 - 6 } & $\mathbf{2 0}$ & $\mathbf{2 5}$ & $\mathbf{3 0}$ & $\mathbf{3 5}$ & $\mathbf{3 8}$ \\
\hline 2.7 & 10.9 & 9.6 & 8.5 & 7.6 & 7.0 \\
\hline 3.0 & 11.3 & 9.8 & 9.0 & 8.2 & 8.0 \\
\hline 3.4 & 11.6 & 10.6 & 9.7 & 8.8 & 8.0 \\
\hline 3.7 & 12.0 & 11.3 & 10.3 & 9.4 & 8.8 \\
\hline 4.0 & 12.5 & 11.9 & 10.8 & 9.4 & 8.8 \\
\hline 5.0 & 13.4 & 12.4 & 10.8 & 9.4 & 8.8 \\
\hline
\end{tabular}

Asimismo, los resultados indican que existen variaciones notables en la cohesión necesaria para disponer de un $\mathrm{FS}=1.2$ cuando la diferencia en la excavación es de un metro de ancho. Es decir, la diferencia existente entre ejecutar los bataches de manera tradicional con esperas y hacerlo con el método planteado en este trabajo, con juntas de conexión (Figura 3b, Tabla 1).

\section{ANÁLISIS Y DISCUSIÓN DE LOS RESULTADOS}

El presente estudio se ha realizado para unas situaciones constructivas concretas en las que se considera que el nivel freático se sitúa por debajo de la cota inferior de la excavación, no existen cargas en coronación y las acciones son exclusivamente estáticas.

Por otra parte, y a pesar de que el procedimiento constructivo es rápido en condiciones normales, en los modelos se han considerado parámetros efectivos (c' y $\Phi$ ') debido a la composición granular y la elevada permeabilidad de estos suelos.

En los modelos, la rotura se produce de forma similar a la realmente observada en obra, produciéndose el fallo de forma poco profunda en el sentido horizontal y sin llegar al pie del talud de excavación (Figura 4).

No obstante, a medida que se aumenta el ancho del batache excavado, aumenta también la profundidad horizontal de la

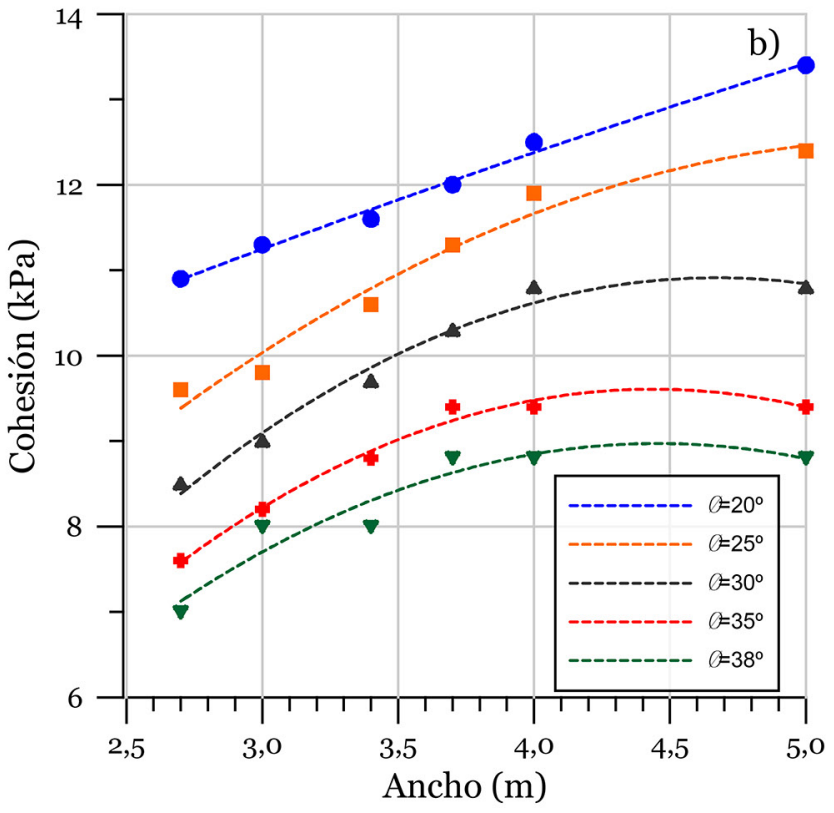

Figura 3. Análisis de la variación de la cohesión necesaria para obtener un FS=1.2 en función del ángulo de fricción para un ancho de batache determinado (a) y tendencia que exhibe la cohesión necesaria para ese mismo FS a medida que aumenta el ancho de batache para cada uno de los valores de ángulos de rozamiento estudiados (b). 
superficie de rotura, pasando de $0.4 \mathrm{~m}$ para los bataches de $2.7 \mathrm{~m}$ de ancho a $1.0 \mathrm{~m}$ para los bataches de $5 \mathrm{~m}$ de ancho. Por esta razón, en los bataches pequeños, si se produce el fallo, éste es menos importante y peligroso, pues es superficial y moviliza una menor masa de suelo (Figura 5).

Además, si se analiza la variación de la cohesión necesaria para obtener un $\mathrm{FS}=1.2$ teniendo en cuenta la diferencia de ancho de excavación de un metro, es decir, 2.7-3.7, 3.0-4.o y 4.0-5.0 m, se observa que esta variación es lineal y constante, adoptando un valor del 10\% para terrenos con un ángulo de rozamiento interno de $20^{\circ}$. Sin embargo, para aquellos suelos con ángulos de rozamiento de $25^{\circ}, 30^{\circ}$ y $35^{\circ}$ esta variación es del orden del 20\% para diferencias de ancho de 2.73.7 y 3.0-4.0, y muy baja o inexistente para la de 4.0-5.0 m. Por tanto, el "efecto batache" se ve claramente disminuido al aumentar la excavación más allá de los cuatro metros. Por último, para terrenos con $\Phi=38^{\circ}$, la variación oscila entre el $27 \%$ para la primera comparación, el $10 \%$ para la segunda y ninguna variación para la tercera.

Finalmente, es importante comentar que la variación entre los valores mínimos y máximos de la cohesión necesarios para obtener un FS $=1.2$ es aproximadamente del 100\% en algunos casos (e.g. 7.0 kPa a $13.4 \mathrm{kPa}$ ). Sin embargo, la diferencia en términos absolutos no es muy significativa principalmente teniendo en cuenta la dificultad que existe en el establecimiento de un valor realista para este parámetro.

\section{CONCLUSIONES}

De los resultados de este estudio se desprende que, para cada uno de los anchos de batache estudiados, la variación de la cohesión para contar con un $\mathrm{FS}=1.2$, a medida que disminuye el ángulo de rozamiento interno, se produce de forma lineal y con un valor de la pendiente muy similar para los anchos de batache 2.7 , 3.0, 3.4, 3.7 y $4.0 \mathrm{~m}$, aumentando considerablemente para el batache de $5 \mathrm{~m}$ de ancho.

Asimismo, comparando la cohesión con el ángulo de rozamiento, ésta varía en función del ancho de batache, exhibiendo una tendencia lineal y creciente para los terrenos con menor ángulo de rozamiento (i.e. $20^{\circ}$ ). Sin embargo, para los terrenos con mayor ángulo de fricción (i.e. 30,35 y $38^{\circ}$ ) esta tendencia se suaviza para el batache de mayor ancho, por lo
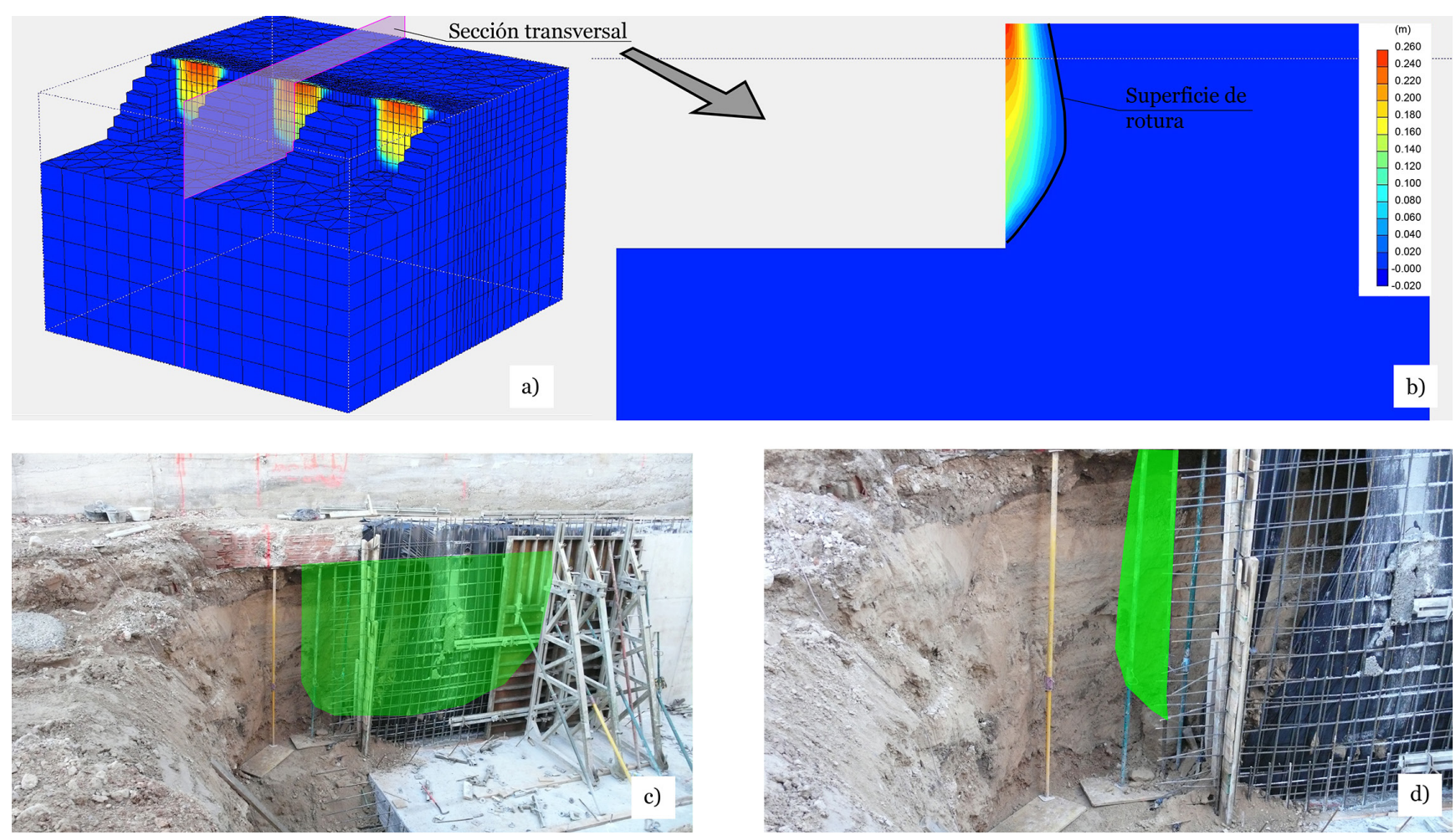

Figura 4. Modelo de elementos finitos 3D en rotura (a) y sección transversal por el plano medio del batache central (b). La gradación de colores representa los desplazamientos totales producidos (m). Rotura real de un batache excavado: frente afectado (c) y profundidad horizontal del material removilizado (d). Las zonas sombreadas en verde delimitan las porciones de batache desestabilizas.
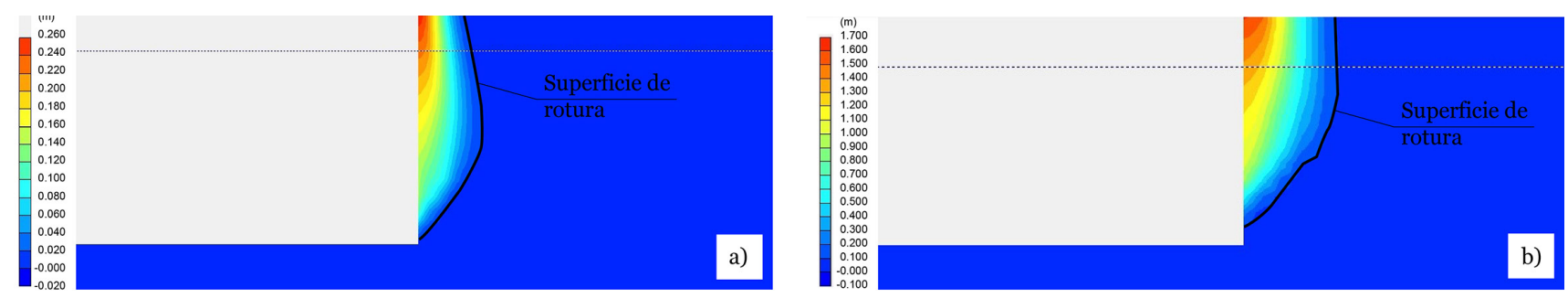

Figura 5. Diferencia en la morfología de la superficie de rotura al aumentar el ancho de los bataches: a) 2.7 m y b) 5.0 m. La gradación de colores representa los desplazamientos totales producidos (m). 
que la tendencia general exhibida es cuadrática, mientras que para terrenos con $\Phi=25^{\circ}$, esta variación es intermedia entre estas dos tendencias descritas.

Por otra parte, mediante esta tipología constructiva los paneles quedan machihembrados por la propia morfología de la junta y la ejecución de la viga de coronación permite que todos los paneles trabajen solidariamente, todo ello sin la necesidad de realizar solapes de armadura. Lo cual permite, además, fijar un cordón hidrófilo sobre la superficie de una de las caras de la junta, que, junto a su geometría, dificultará una hipotética entrada de agua durante la vida útil del edificio.

Además de las ventajas constructivas expuestas, queda patente el aumento de la seguridad al ejecutar los bataches con la técnica descrita en este trabajo. Según este estudio, la disminución en un metro el ancho de excavación repercute en la mayoría de los casos, en un descenso de al menos un 10\% de la cohesión necesaria para mantener un $\mathrm{FS}=1.2$, manteniendo el ancho de muro realmente ejecutado.

Por último, cabe destacar que los gráficos propuestos en este trabajo (Figura 3) permitirán diseñar de forma óptima bataches excavados en terrenos mixtos de características similares a los considerados en este estudio con una seguridad suficiente para garantizar su estabilidad temporal.

\section{AGRADECIMIENTOS}

Este trabajo ha llevado a cabo durante la estancia del primer autor en la Universidade do Minho (Portugal) en el marco del proyecto CAS17/o0392, financiado por el Ministerio de Educación Cultura y Deporte del Gobierno de España.

Asimismo, queremos agradecer a la Empresa INGYOB, S.L., la cesión de alguna de las fotografías insertadas en las figuras de este trabajo.

\section{REFERENCIAS}

(1) Ministerio de la Vivienda (2008). Código Técnico de la Edificación. Documento Básico - Seguridad Estructural: Cimientos (CTE SE-C), Madrid, España.

(2) Hilsey, A.E. (1981). Multi-purpose precast concrete panels, and methods constructing concrete structures employing the same. United States Patent 4,290,246. 22 pp. Retreived from http://www.freepatentsonline.com/4290246.html

(3) Ministerio de la Vivienda (1976). ORDEN de 1 marzo 1976, Norma Tecnológica de la Edificación NTE-ADV/1976, «Acondicionamiento del terreno. Desmontes: Vaciado» BOE 6 marzo 1976 y 13 marzo 1976, núm. 57 y 63.

(4) BSI British Standards, 2009. BSI BS 6031:2009 Code of practice for earthworks. Civil Engineering and Building Structures Standards Committee. Retrieved from https://geotechnicaldesign.info/bs6031-2009.html

(5) Clough, G.W., Denby, G.M. (1977). Stabilizing berm design for temporary walls in clay. Journal of the Geotechnical Engineering Division, 103(2): 75-90.

(6) Powrie, W., Davies, J.N., Britto, A.M. (1992, 20-23 july). A cantilever retaining wall supported by a berm during temporary works activities. In ICE conference on retaining structures. Cambridge: Robinson College, 418-428.

(7) Gourvenec, S.M., Powrie, W. (2000). Three-dimensional finite element analyses of embedded retaining walls supported by discontinuous earth berms. Canadian Geotechnical Journal, 37(5): 1062-1077. https://doi.org/10.1139/too-033

(8) Gomaa, Y. (2003). Berms for stablizing earth retaining structures. Master thesis. Faculty of Engineering, Cairo University.

(9) Liao, H.J., Lin, C.C. (2009). Case studies on bermed excavation in Taipei silty soil. Canadian Geotechnical Journal, 46(8): 889-902. https://doi.org/10.1139/To9-034

(10) Ng, C.W.W., Lei, G.H. (2003). An explicit analytical solution for calculating horizontal stress changes and displacement around an excavated diaphragm wall panel. Canadian Geotechnical Journal, 40(4): 780-792. https://doi.org/10.1139/ to3-027

(11) Gourvenec, S.M., Powrie, W. (1999). Three-dimensional finite element analysis of diaphragm wall installation. Géotechnique, 49(6): 801-823. https://doi.org/10.1680/geot.1999.49.6.801

(12) Lei, G.H., Ng, C.W.W., and Rigby, D.B. (2001). Stress and displacement around an elastic artificial rectangular hole. Journal of Engineering Mechanics, ASCE, 127(9): 880-890. https://doi.org/10.1061/(ASCE)0733-9399(2001)127:9(880)

(13) Soroush, A., Soltani-Jigheh, H. (2009) Pre- and post-cyclic behavior of mixed clayey soils. Canadian Geotechnical Journal, 46(2): 115-128. https://doi.org/10.1139/To8-109

(14) Tsotsos, S., Karaoulanis, F.E. \& Chatzigogos, T. (2010). A New Concept on the Compressibility of Mixed Soils: Experimental and Numerical Approach. Geotechnical and Geological Engineering, 28: 199-207. https://doi.org/10.1007/ s10706-009-9283-y

(15) Cruz, N., Rodrigues, C., da Fonseca, A. (2012). Design parameters of Portuguese granitic residual soils obtained from DMT tests. International Journal of Geotechnical Engineering, 6(2): 239-244. https://doi.org/10.3328/ IJGE.2012.06.02.239-244

(16) Viana da Fonseca, A., Matos Fernandes, M., Cardoso, A.S., Barreiros Martins, J. (1994). Portuguese experience on geotechnical characterization of residual soils from granite. In Proc. XIII ICSMFE, New Dehli, India, Janeiro, Vol. 1, pp. 377-380. Rotterdam: A.A. Balkema.

(17) Cruz, N., Viana da Fonseca, A., Neves, E. (2004). Evaluation of effective cohesive intercept on residual soils by DMT data. Geotechnical and Geophysical Site Characterization. Proc. 2nd Int. Site Characterization - ISC'2, Porto, Portugal, Sept. 2004. Rotterdam: Millpress.

(18) Cruz, N. (2010). Modelling Geomechanics of Residual Soils with DMT Tests. PhD thesis. Porto: Porto University.

(19) Zienkiewicz, O.C., Humpheson, C., Lewis, R.W. (1975). Associated and non-associated visco plasticity and plasticity in soil mechanics. Géotechnique, 25(4): 671-689. https://doi.org/10.168o/geot.1975.25.4.671

(20) Ministerio de Fomento (2009). Guía de Cimentaciones en Obras de Carretera. Madrid: Ministerio de Fomento. 
(21) Orr, T. (2013). Slope stability design of slopes and embankments. JRC Report: Eurocode 7: Geotechnical Design - Worked examples. European Commission. 13-14 June 2013, Dublin Ireland. Retrieved from http://eurocodes.jrc.ec.europa. eu/showpage.php?id=2013_o6_WS_GEO

(22) Orr, T. (2005). Proceedings of the International Workshop on the Evaluation of Eurocode 7. Dublin, Ireland: The Department of Civil, Structural and Environmental Engineering, Trinity College..

(23) American Association of State Highway and Transportation Officials (2014). AASHTO LRFD Bridge Design Specifications. Washington.

(24) Naval Facilities Engineering Command (1986). Soil Mechanics DESIGN MANUAL 7.01. Virginia.

(25) Laboratorio Oficial J. M. Madariaga (LOM) (2015). Guía sobre control geotécnico en minería a cielo abierto. Madrid: Dirección General de Política Energética y Minas del Ministerio de Industria, Energía y Turismo.

(26) Lees A. (2012). Obtaining Parameters for Geotechnical Analysis, p. 7. Reino Unido: NAFEMS.

(27) Potts D.M., Zdravkovic L. (2001). Finite element analysis in geotechnical engineering: application. p. 126. London: Thomas Teldord.

(28) Segato, D., Scarpelli G. (2006). Morphological effects on settlements induced by shallow tunneling. In Proceedings of the sixth European conference on Numerical Methods in Geotechnical Engineering. London: Schweiger, Taylor and Francis Group. 